\title{
Aprendizagem colaborativa como ferramenta no ensino de Anatomia Humana
}

\author{
Collaborative learning as a tool in the teaching of Human Anatomy \\ El aprendizaje colaborativo como herramienta en la enseñanza de la Anatomía Humana
}

Recebido: 14/03/2021 | Revisado: 20/03/2021 | Aceito: 24/03/2021 | Publicado: 02/04/2021

Sarah Lima Campos

ORCID: https://orcid.org/0000-0002-9302-1606 Universidade Federal do Tocantins, Brasil E-mail: sarahlimacampos14@gmail.com

Ana Kleiber Pessoa Borges

ORCID: https://orcid.org/0000-0003-1865-2595 Universidade Federal do Tocantins, Brasil E-mail: anakleiber@uft.edu.br

Tales Alexandre Aversi-Ferreira

ORCID: https://orcid.org/0000-0002-4417-7209 Universidade Federal de Alfenas, Brasil

E-mail: aversiferreira@gmail.com

Andressa Diúlia Dantas da Silva

ORCID: https://orcid.org/0000-0003-2099-5669

Universidade Federal do Tocantins, Brasil

E-mail: andressa.diulia10@gmail.com

Aline Lima Campos

ORCID: https://orcid.org/0000-0003-3239-692X

Centro Universitário Faveni, Brasil

E-mail: alinecamposeng@ hotmail.com

Tainá de Abreu

ORCID: https://orcid.org/0000-0001-5043-7014

Universidade Federal do Tocantins, Brasil

E-mail: tainaabreu@mail.uft.edu.br

Walesca Rodrigues Silva

ORCID: https://orcid.org/0000-0003-1328-2222

Universidade Federal do Tocantins, Brasil

E-mail: walesca.rodrigues@mail.uft.edu.br

\begin{abstract}
Resumo
Os conhecimentos anatômicos são imprescindíveis para o profissional da área de saúde, não obstante essa disciplina apresenta baixos desempenhos em relação ao processo de ensino e aprendizagem. Com isso esta pesquisa teve por objetivo propor o uso da aprendizagem colaborativa como ferramenta de auxílio ao aprendizado de anatomia. O método foi aplicado na forma de monitorias para os discentes dos cursos de Nutrição e Enfermagem da Universidade Federal do Tocantins (UFT), campus de Palmas-TO. Os discentes foram divididos em dois grupos, A e B, um grupo era responsável por abordar o conteúdo teórico e o outro pela explanação e condução do conteúdo prático, havendo rodízio destas atribuições. A eficácia do método foi avaliada por meio de questionário que empregou a escala de Likert e análise do rendimento dos discentes, através do diário de notas da disciplina. Para síntese dos resultados foi aplicado o Teste de Tukey. Observou-se que 95\% dos participantes após a participação da metodologia afirmaram ter obtitido uma evolução positiva em relação ao aprendizado da anatomia humana e recomendaram a aplicação do método em outras disciplinas. Tendo o grupo de discentes participantes da metodologia colaborativa apresentado resultados superiores à média e ao grupo de discentes não participantes, com HSD (Diferença Honestamente Significativa) de 5\%. Diante dos grandes desafios enfrentados no ensino de anatomia, o estímulo para execução da monitoria na forma colaborativa pode ser uma ferramenta para auxiliar nas estratégias de ensino.
\end{abstract}

Palavras-chave: Aprendizagem colaborativa; Anatomia humana; Ensino de anatomia; Aprendizagem em anatomia; Ensino.

\begin{abstract}
Anatomical knowledge is essential for health professionals, although this discipline presents low performances in relation to the teaching and learning process. Thus, this research aimed to propose the use of collaborative learning as a tool to aid anatomy learning. The method was applied in the form of monitoring for students of the Nutrition and Nursing courses at the Federal University of Tocantins (UFT), campus of Palmas-TO. The students were divided into two groups, A and B, one group was responsible for addressing the theoretical content and the other for explaining and conducting the practical content, with rotation of these assignments. The effectiveness of the method was assessed
\end{abstract}


by means of a questionnaire that used the Likert scale and analysis of the students' performance, through the notes diary of the discipline. To synthesize the results, the Tukey test was applied. It was observed that $95 \%$ of the participants after participating in the methodology stated that they had obtained a positive evolution in relation to the learning of human anatomy and recommended the application of the method in other disciplines. As the group of students participating in the collaborative methodology presented results above the average and the group of nonparticipating students, with HSD (Honestly Significant Difference) of 5\%. In view of the great challenges faced in the teaching of anatomy, the encouragement to perform monitoring in a collaborative way can be a tool to assist in teaching strategies.

Keywords: Collaborative learning; Human anatomy; Anatomy teaching; Anatomy learning; Teaching.

\section{Resumen}

El conocimiento anatómico es fundamental para los profesionales de la salud, aunque esta disciplina presenta bajos rendimientos en relación al proceso de enseñanza y aprendizaje. Por lo tanto, esta investigación tuvo como objetivo proponer el uso del aprendizaje colaborativo como una herramienta para ayudar al aprendizaje de la anatomía. El método se aplicó en forma de seguimiento a estudiantes de los cursos de Nutrición y Enfermería de la Universidad Federal de Tocantins (UFT), campus de Palmas-TO. Los alumnos se dividieron en dos grupos, A y B, un grupo se encargaba de abordar los contenidos teóricos y el otro de explicar y conducir los contenidos prácticos, con rotación de estos trabajos. La efectividad del método se evaluó mediante un cuestionario que utilizó la escala Likert y el análisis del desempeño de los estudiantes, a través del diario de apuntes de la disciplina. Para sintetizar los resultados se aplicó la prueba de Tukey. Se observó que el $95 \%$ de los participantes luego de participar en la metodología manifestaron haber obtenido una evolución positiva en relación al aprendizaje de la anatomía humana y recomendaron la aplicación del método en otras disciplinas. Como el grupo de estudiantes participantes en la metodología colaborativa presentó resultados por encima de la media y el grupo de estudiantes no participantes, con HSD (Honestly Significant Difference) del 5\%. En vista de los grandes desafíos que enfrenta la enseñanza de anatomía, el estímulo para realizar el monitoreo de manera colaborativa puede ser una herramienta para ayudar en las estrategias de enseñanza.

Palabras clave: Aprendizaje colaborativo; Anatomía humana; Enseñanza de la anatomía; Aprendizaje de anatomia; Ensenãnza.

\section{Introdução}

A anatomia, palavra derivada do grego anatome (ana $=$ em partes, tome corte) é o principal componente curricular presente nos ciclos básicos dos cursos relacionados com as ciências da saúde (Gardner, Gray \& O'rahilly, 1963; Sugand, Abrahams \& Khurana, 2010). Seu principal objeto de estudo é a compreensão do corpo humano, no qual descreve suas estruturas em relação à forma, localização e relações (Standring, 2005).

No âmbito acadêmico é um componente curricular presente nos períodos iniciais e que permite o primeiro contato dos futuros profissionais com o corpo humano e também a construção de um alicerce para a formação clínica e específica (Mclachlan \& Patten, 2006). Apesar de se conhecer toda a importância que a anatomia apresenta na formação dos profissionais da saúde, esse é um dos componentes curriculares que possui um dos mais baixos desempenhos em relação ao processo de ensino e aprendizagem e que pode colaborar para a evasão acadêmica logo nas séries iniciais dos cursos (Almeida et al., 2015). Os principais motivos apontados são as reduções nas cargas horárias da disciplina, diminuição das práticas de dissecação, uso de metodologias de ensino inapropriadas (Pfrimer et al., 2012, Mclachlan \& Patten, 2006; Nóbrega \& Tavares, 2008; AversiFerreira et al., 2017).

Assim, o estudo dos métodos no ensino de anatomia tem sido amplamente debatido, (Moxham \& Moxham, 2007;

Patel \& Moxham, 2008; Kerby \& Shukur, 2011) como por exemplo, o uso body painting (pintura corporal), Aprendizag em Baseada em Problemas (PBL- Problem Based Learning), aprendizagem baseada em equipes (TBL - Team Based Learning), Sala de aula invertida com uso de tecnologias (vídeos de anatomia tridimensional em 3D) (Tiznado-Matzner, G., BucareyArriagada, \& Lizama-Pérez, 2020), construção de materiais tridimensionais (De Castro et al. 2018 ), uso de peças anatômicas em resina, plastinação, práticas de dissecação e também o método tradicional, que é centrada no professor.

Na maioria das universidades, o ensino de anatomia consiste em combinação de palestras e sessões de laboratório (Bergman et al., 2013), com predominância do método de ensino tradicional, que muitas vezes é marcado por um sistema 
baseado em decorar estruturas e com poucas correlações entre as próprias estruturas estudadas ou até mesmo com as demais disciplinas. Tendo em vista esse cenário tradicional com as novas possibilidades para fortalecer os processos de ensinoaprendizagem, o ensino de anatomia precisa ser constantemente revisto e analisado para uma melhor formação acadêmica dos alunos (Sugand, Abrahams \& Khurana, 2010).

Observa-se também problemas estruturais enfrentados pelos docentes no ensino de anatomia como a redução de carga horária, diminuição ou exclusão das práticas de dissecação, alto custo para manutenção e falta de profissionais capacitados para cuidar dos laboratórios (Mclachlan \& Patten, 2006; Leung et al., 2006; Nóbrega \& Tavares, 2008; Aversi-Ferreira et al., 2017), além da diminuição de cadáveres recebidos pelas instituições, questão essa que pode ser contornada por meio dos programas de doação de corpos (Da Silva et al., 2020). Body Donation Programs in Brazil. Brazilian Journal of Development, 6(12), 101260-101271.). Em relação aos discentes, as maiores dificuldades estão relacionadas à memorização das estruturas, o preparo inadequado das peças para estudo, e principalmente, as técnicas e métodos de ensino adotadas pelos docentes (Braz, 2009; Carvalho, 2017).

Devido à grande complexidade no processo de ensino-aprendizagem da anatomia e considerando a grande quantidade e detalhamento dos conteúdos envolvidos (Salbego et al., 2015) e as novas possibilidades tecnológicas que podem ser usadas como ferramentas para facilitação do ensino é importante que os métodos de ensino precisam ser repensados, a fim de corresponder às expectativas deste novo contexto educacional (Fornaziero et al., 2010; Tanasi, Tanase \& Harsovescu, 2014).

Bellan (2008) acredita que instituições educacionais necessitam de uma aprendizagem rápida e flexível que permita desenvolver a capacidade de aprender por si mesmo e aprimorar as habilidades pessoais de seus colaboradores. Segundo Hase \& Kenyon (2011) não se trata diretamente da relação ensino-aprendizagem, mas o foco se desloca para uma aprendizagem que seja auto determinada pelo aluno, ele é quem define como e quando aprender. Isso representa o evoluir de um discente com consciência ingênua para um discente com consciência crítica, o que requer uma postura ativa com curiosidade criativa, questionadora e sempre insatisfeita (Mitre et al., 2008). A aprendizagem é um processo construtivo na qual os alunos conectam novas informações a uma rede de conhecimento, formando e fortalecendo conexões significativas entre conceitos (Regehr \& Norhomem, 1996).

Ao praticar este tipo de construção de conhecimento trabalha-se com a visão da psicologia cognitiva que tem como base norteadora a teoria cognitivista de David Ausubel, a qual objetiva-se na construção de uma aprendizagem significativa tomando por base os conhecimentos prévios dos discentes e tornando-os agentes no processo de ensino-aprendizagem. Entende-se que cada um tem, a partir de sua própria experiência, conhecimento sobre vários aspectos formados em sua própria consciência. Segundo Ausubel (2013) o que ainda está por fazer em termos de investigação e de educação é: mais investigação sobre a aquisição e a retenção de áreas de estudo completas e de currículos de forma sequencial para diferentes faixas etárias de estudantes; e a aplicação de tais resultados ao currículo e às práticas e materiais de instrução.

Embora haja abundância de informações disponíveis sobre o potencial das contribuições das teorias de aprendizagem, existem poucos relatos de teorias de aprendizagem sendo utilizadas para resolver problemas práticos de aprendizagem (Ertmer \& Newby, 1993). É certo que os tempos estão mudando e o ensino universitário não está isento das repercussões na sociedade que ocorrerem como resultado do desenvolvimento e utilização de novos tecnologias (Pereira, Schmitt \& Dias, 2007). E a educação é uma forma de intervenção no mundo que vai além dos conhecimentos dos conteúdos ensinados e/ou aprendidos (Souza Júnior et al., 2010), nesse contexto a anatomia tem que ser vista como um assunto tridimensional que requer uma compreensão sólida das relações entre as estruturas (Longhurst et al., 2020).

Por fim o conhecimento neste componente curricular vai ser útil não somente na graduação, que dura alguns anos, mas também na vida profissional. E é neste paradigma que Mitre (et al., 2008) acredita ser essencial pensarem uma metodologia para uma prática de educação libertadora, na formação de um profissional ativo, apto a aprender a aprender. Já se 
colocou a hipótese de que todas as experiências de aprendizagem passadas influenciam, ou exercem efeitos positivos ou negativos sobre, a nova aprendizagem significativa, devido ao impacto sobre as propriedades relevantes da estrutura cognitiva (Ausubel, 2003).

Este trabalho busca subsídios dentro do contexto do ensino com métodos ativos, mais especificamente no uso da aprendizagem colaborativa, para analisar a contribuição deste na aprendizagem significativa e redução das dificuldades enfrentadas pelos discentes que cursaram Anatomia Humana nos cursos de Enfermagem e Nutrição na Universidade Federal do Tocantins (UFT), campus Palmas. O uso de metodologias colaborativas definida por Gokhale (1995), é um método de instrução, no qual os alunos trabalham em grupos e em direção a um objetivo acadêmico comum. Essa metodologia colaborativa pode ser aplicada no ensino de anatomia como uma alternativa para minimizar as dificuldades já mencionadas. Partindo desta explanação, este trabalho levanta o seguinte problema: pode a aprendizagem colaborativa provocar mudança de paradigmas em relação ao ensino e aprendizagem em anatomia humana e ser uma ferramenta eficaz para a melhoria da qualidade de ensino nesse componente curricular?

\section{Metodologia}

Pesquisa aplicada com estudo descritivo, exploratório e indutivo, de abordagem quali/quantitativas, realizada na Universidade Federal do Tocantins (UFT), campus Palmas que objetiva propor o uso de metodologias interativas, como a aprendizagem colaborativa, no ensino de anatomia humana para o auxílio no aprendizado. A presente pesquisa foi aprovada pelo Comitê de Ética do Instituto Federal de Educação, Ciência e Tecnologia do Tocantins - IFTO, Campus Palmas, com o número do CAEE 22749019.3.0000.8111.

A população investigada foi composta por discentes do primeiro período, entre 2017 e 2018 dos cursos de Nutrição e Enfermagem da Universidade Federal do Tocantins (UFT), campus Palmas. Os critérios de delineamento amostral foram os alunos estarem matriculados na disciplina de Estudos Morfológicos Macroscópicos (Anatomia Humana), maiores de dezoito anos, serem frequentes nas monitorias e apresentarem o termo de consentimento livre e esclarecido assinado. A amostra foi escolhida através do método de amostragem por conveniência, com participação nas monitorias de forma facultativa, submetendo ao questionário somente os discentes que participaram ativamente das monitorias na modalidade de aprendizagem colaborativa. Assim foi considerado o método não probabilístico por quota, frequentemente utilizado em pesquisas exploratórias (Schiffman \& Kanuk, 2000).

O método de avaliação se deu em duas fases. A primeira fase foi a exploração qualitativa por meio de questionário semiestruturado (Tabela 1) com perguntas subjetivas e objetivas aplicadas após o encerramento de semestre letivo. A segunda fase investigou o rendimento dos alunos na forma quantitativa através do cálculo de médias e medidas de variação, como o desvio padrão, variância, coeficiente de variação e aplicação de teste estatístico de comparação de resultados. 
Tabela 1 - Questionário semiestruturado.

1. Após a monitoria na forma colaborativa tive uma evolução positiva sobre minha aprendizagem em anatomia humana

2. Durante a monitoria consegui associar melhor o conteúdo teórico com o prático

3. Depois da experiência com a monitoria passei a buscar outras fontes de informação além das sugeridas pelo professo

4. Estudei previamente os conteúdos antes da monitoria

5. A monitoria dessa forma colaborativa contribuiu para minha habilidade em sintetizar/resumir o conteúdo

6. Antes das monitorias eu tinha dificuldade em estudar a anatomia

7. Durante a monitoria me senti mais motivada para estudar

8. Após as monitorias observei melhora na minha oratória

9. Antes da monitoria eu não tinha uma interação com a minha turma

10. Após a monitoria posso dizer que melhorei minha relação com os meus colegas

11. Essa forma de monitoria despertou meu desejo pela docência

12. Durante a monitoria eu sentia satisfação por transmitir os conteúdos aos colegas

13. Participava das monitorias por apenas temer a reprovação

14. Após a monitoria passei a usar mapas mentais, esquemas, resumos, desenhos e/ou fluxograma para reforçar meu aprendizado

15. Após a monitoria aprendi a planejar melhor meu estudo

16. Antes das monitorias eu não tinha um planejamento de estudo

17. Observei que discutir as dificuldades em grupo é uma forma eficaz de praticar o que eu aprendi

18. Durante a monitoria pude contribuir ativamente com as discussões em grupo

19. Durante a monitoria o grupo trabalhou bem em conjunto

20. Durante a monitoria usei os meios eletrônicos com eficiência como fonte de estudo

21. Eu recomendaria que esse método de monitoria colaborativa fosse aplicado em outros componentes curriculares (disciplinas)

22. Após as monitorias aprendi a trabalhar em grupo e/ou equipe

23. Após a monitoria consegui ter melhor desempenho nas avaliações

24. Posso dizer que a monitoria executada na forma colaborativo despertou em mim:

Fonte: Autores.

Para minimizar os riscos o questionário foi aplicado de forma individual, plataforma Google Forms. Durante o período de coleta de dados foram convidados os alunos matriculados entre 2017 e 2018 nos cursos de nutrição e enfermagem, totalizando 194 discentes. Destes 85 aceitaram participar e apenas 34 responderam o questionário.

Num segundo momento foi analisado o rendimento acadêmico dos 194 alunos, tendo sido feito um comparativo entre os que não participaram das monitorias ativas contra os que participara. Portanto, a amostra final deste estudo foi de 194 discentes (Tabela 2), sendo 129 do curso de nutrição (66\%) e 65 do curso de enfermagem (34\%). 
Tabela 2 - Relação curso x ano x quantitativo de participantes.

\begin{tabular}{|c|c|c|c|c|}
\hline \multirow[t]{2}{*}{ TURMA } & \multicolumn{2}{|c|}{ NUTRIÇÃO } & \multicolumn{2}{|c|}{ ENFERMAGEM } \\
\hline & Matriculados & Participantes da monitoria & Matriculados & Participantes da monitoria \\
\hline $2017 / 1$ & 43 & 16 & 16 & 8 \\
\hline $2017 / 2$ & 44 & 16 & 19 & 11 \\
\hline $2018 / 1$ & 42 & 17 & 30 & 17 \\
\hline TOTAL & 129 & 49 & 65 & 36 \\
\hline
\end{tabular}

Fonte: Dados Trabalhados pelos autores.

Os questionários aplicados foram respondidos por cada participante somente uma vez, sendo cadastrado o e-mail do discente entrevistado na plataforma do google forms, de forma a evitar duplicidade de dados. As afirmativas do questionário foram embasadas nas estratégias de autorregulação da aprendizagem, conforme colocado por Da Veiga Simão e Frison (2016) as estratégias cognitivas/metacognitivas, motivacionais e comportamentais empregadas pelos participantes.

1. Estratégias cognitivas /metacognitivas: organizar-se e tomar consciência das demandas exigidas pelas tarefas propostas, resolver as situações desafiadoras, avaliar os procedimentos escolhidos e os resultados alcançados; 2. Estratégias motivacionais: a compreensão das razões para mover esforços para aprender e ajudar a lidar melhor com o sucesso e o fracasso, com as possíveis desmotivações; 3 . Estratégias comportamentais: controlar o tempo, organizar o material e o local de estudo, solicitar ajuda aos mais experientes (Da Veiga Simão \& Frison, 2013).

A aprendizagem autorregulada é a fusão de habilidade e vontade (Montalvo \& Torres, 2004). Os alunos sentem-se agentes do seu comportamento, acreditam que a aprendizagem é um processo proativo, e estão automotivados e usam estratégias que lhes permitem obter os resultados acadêmicos desejados (Da Veiga Simão \& Frison, 2013). Dentro da análise cognitiva e metacognitivas foram abordado os seguintes aspectos (Tabela 3): associação do conteúdo, fontes de estudo, interesse pela docência, uso de estratégias de aprendizagem, interação com pares, aplicabilidade do metodo, entre outros.

Tabela 3 - Afirmativas de abordagem cognitiva/metacognitivas.

\section{Estratégias cognitivas /metacognitivas}

- Durante a monitoria consegui associar melhor o conteúdo teórico com o prático.

- Depois da experiência com a monitoria passei a buscar outras fontes de informação além das sugeridas pelo professor.

- Essa forma de monitoria despertou meu desejo pela docência.

- Após a monitoria passei a usar mapas mentais, esquemas, resumos, desenhos e/ou fluxograma para reforçar meu aprendizado.

- Observei que discutir as dificuldades em grupo é uma forma eficaz de praticar o que eu aprendi.

- Durante a monitoria pude contribuir ativamente com as discussões em grupo.

- Eu recomendaria que esse método de monitoria colaborativa fosse aplicado em outros componentes curriculares (disciplinas).

- Após a monitoria consegui ter melhor desempenho nas avaliações

Fonte: Dados Trabalhados pelos autores.

Consoantemente na análise motivacional, expresso na Tabela 4, abordou-se a percepção de evolução no aprendizado, dificuldades, motivação, satisfação, medo e interação pessoal com seus pares. Segundo Sousa e Júnior (2017) o aprendiz só verá sentido em utilizar essas estratégias de aprendizagem quando tiver percepção da sua relevância, tendo que as variáveis motivacionais estão diretamente relacionadas ao uso ou não dessas estratégias. É compreensível que o aluno desenvolva emoções no decorrer do processo de aprendizagem, cabe a ele saber controlar e modifica-las, ajustando-as a situação. 
Tabela 4 - Afirmativas de abordagem motivacional.

\section{Estratégias motivacionais}

- Após a monitoria na forma colaborativa tive uma evolução positiva sobre minha aprendizagem em anatomia humana.

- Antes das monitorias eu tinha dificuldade em estudar a anatomia.

- Durante a monitoria me senti mais motivada para estudar.

- Após as monitorias observei melhora na minha oratória.

- Antes da monitoria eu não tinha uma interação com a minha turma.

- Após a monitoria posso dizer que melhorei minha relação com os meus colegas.

- Durante a monitoria eu sentia satisfação por transmitir os conteúdos aos colegas.

- Participava das monitorias por apenas temer a reprovação.

- Durante a monitoria o grupo trabalhou bem em conjunto.

Fonte: Dados Trabalhados pelos autores.

A fim de analisar se houve uma aplicabilidade estratégica comportamental após o metodo ativo, nas afirmativas da Tabela 5 indagamos os alunos sobre planejamento e controle do tempo de estudo, os esforços desempenhados pelos mesmos, a percepção de se formar um ambiente favorável à aprendizagem, bem como procurar ajuda perante as dificuldades.

Tabela 5 - Afirmativas de abordagem comportamental.

\section{Estratégias comportamentais}

- Estudei previamente os conteúdos antes da monitoria.

- A monitoria dessa forma colaborativa contribuiu para minha habilidade em sintetizar/resumir o conteúdo.

- Após a monitoria aprendi a planejar melhor meu estudo.

- Antes das monitorias eu não tinha um planejamento de estudo.

- Durante a monitoria usei os meios eletrônicos com eficiência como fonte de estudo.

- Após as monitorias aprendi a trabalhar em grupo e/ou equipe.

- Posso dizer que a monitoria executada na forma colaborativo despertou em mim

Fonte: Dados Trabalhados pelos autores.

\section{Resultados e Discussão}

Durante o semestre os alunos seguiram com as aulas administradas de forma tradicional, aulas teóricas e práticas, ambas conduzidas pelo mesmo docente. Associada as aulas tradicionais foram administradas monitorias com método ativo baseada na aprendizagem colaborativa, sendo os alunos divididos em dois grupos, A e B. O grupo A em uma semana ficava responsável por abordar o conteúdo teórico e o grupo B responsável pela explanação e condução do conteúdo prático, havendo rodízio destas atribuições. O proposto era que o grupo fizesse explanação do conteúdo e retirasse dúvidas com seus pares.

As tarefas assumidas pelos alunos monitores têm como objetivo auxiliar o professor titular, mas, nos cursos superiores, a monitoria tem sido utilizada, como estratégia de apoio ao ensino, especialmente para atender estudantes com dificuldades de aprendizagem (Da Veiga Simão \& Frison, 2013). Sabe-se que quando o aluno participa da elaboração de material nessas metodologias alternativas, aumentam a compreensão sobre o conteúdo, e a preocupação com detalhes intrínsecos é a melhor forma de representá-los (Souza Júnior et al., 2010).

O conteúdo do estudo ativo seguiu o cronograma das aulas administradas tradicionalmente, com estudo da anatomia sistêmica. Todo os sistemas anatômicos foram abordados em um semestre e ao final deste foi aplicado um questionário a fim de verificar a percepção dos alunos em relação a metodologia colaborativa usada nas monitorias. O meio escolhido para aplicação dos questionários foi o Google forms, que manteve maior privacidade e conforto ao aluno. Adicionalmente foi analisado o rendimento acadêmico pelas notas adquiridas nas avaliações aplicadas no decorrer do semestre.

A pesquisa empregou a escala de Likert de cinco pontos, com a seguinte análise: 1 = Concordo totalmente: concorda em 100\% da afirmativa; 2 = Concordo parcialmente: concorda com a afirmativa, mas não em 100\%; 3 = Sem condições de 
opinar; 4 = Discordo parcialmente: discorda da afirmativa, mas não em 100\%; 5 = Discordo totalmente: discorda em $100 \%$ da afirmativa. Likert é empregada em pesquisas de opinião, na qual os entrevistados especificam o seu nível de concordância com a afirmação.

Formulou-se então um questionário de 23 afirmações com base na escala Likert e uma pergunta subjetiva, ambas baseadas na aprendizagem autorreguladora, atentando para abordar as estratégias cognitivas/metacognitivas, motivacional e comportamental sofridas pelos alunos. O questionário foi aplicado somente aos participantes da monitoria $(\mathrm{N}=85)$, contudo houve adesão de respostas de apenas 43,52\% dos participantes. Fato decorrente da aplicação do questionário ter sido no período de férias, ou seja, período não letivo dos alunos.

Gráfico 1 - Relação percentual das respostas das afirmativas.

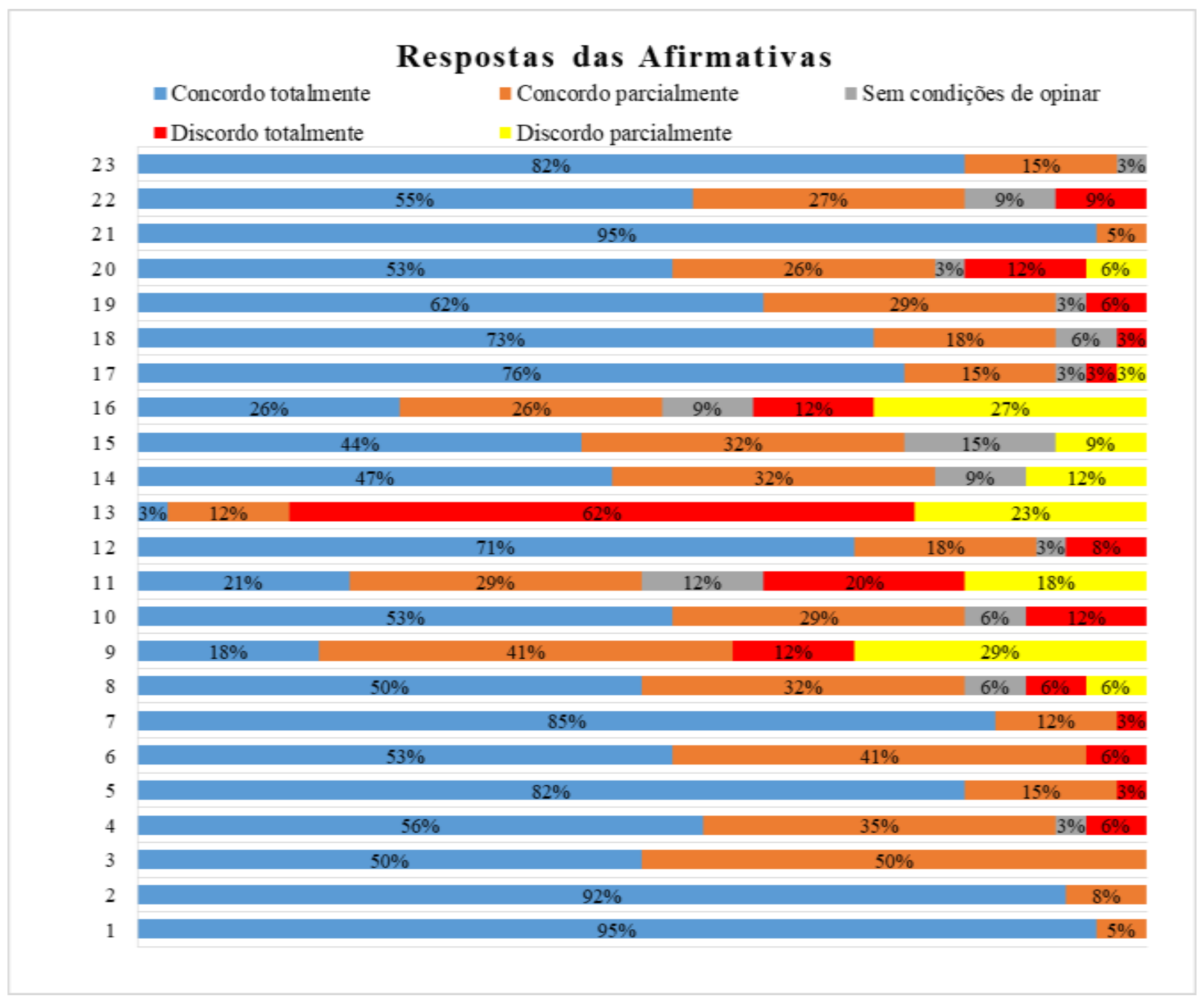

Fonte: Dados Trabalhados pelos autores.

Através do gráfico é possível verificar que todas as afirmativas, com exceção das questões 9, 11, 13, 14, 15 e 16, apresentaram aceitabilidade (concordo totalmente) acima de 50\%. A maioria dos discentes, cerca de $95 \%$, após a participação da metodologia colaborativa afirmam que obtiveram uma evolução positiva em relação ao aprendizado da anatomia humana e recomendariam a aplicação do método em outras disciplinas. 
Dentre as questões com aceitabilidade, concordo totalmente, inferior a 50\%, tem-se afirmativas correspondente ao aspecto motivacional, a exemplo das questões 9 e 13. Na afirmativa 09 (Antes da monitoria eu não tinha uma interação com a minha turma) $41 \%$ dos entrevistados concordaram parcialmente e $29 \%$ discordaram parcialmente, isso confirma que independente da monitoria na forma colaborativa, que visava o ensino entre pares, já exista uma interação social em sala de aula, aspecto visto como positivo, que retrata sobre a zona de desenvolvimento proximal, onde a interferência de outros indivíduos é mais transformadora nas relações entre desenvolvimento e aprendizado (Vygotsky, 2012).

Já afirmativa 13 (Participava da monitoria por apenas temer a reprovação) apresenta-se como a questão com menor percentual de aceitabilidade, tendo apenas um percentual de $3 \%$ dos alunos concordado totalmente com a afirmação e a maioria dos discentes (62\%) discordaram totalmente, fato positivo que demonstra que os participantes da monitoria a fizeram por interesse e busca própria, não pelo fato de sentir-se pressionado ou por estarem preocupados com a aquisição de nota para aprovação no componente curricular.

Vinculado ao grupo de questões com baixa aceitabilidade, têm-se as de aspectos cognitivos/metacognitivos que são as afirmativas 11 e 14. Na afirmativa 11 (Essa monitoria despertou em mim o desejo pela docência) temos $21 \%$ concordando totalmente. Estes perceberam a monitoria como uma iniciação à docência, tendo assim atingido um dos objetivo esperado da pesquisa que era o despertar para docência de forma a ampliar a visão do monitor sobre a vida acadêmica, aproximando-lhe do tripé universitário: ensino, pesquisa e extensão. Contrateve-se $20 \%$ discordando totalmente, estes não viram a monitoria como um despertar para docência. Todavia, a monitoria possibilitou a estes um enriquecimento curricular, experiência e uma participação ativa no processo de construção de conhecimento.

Já na afirmativa 14 (Após a monitoria passei a usar mapas mentais, esquemas, resumos, desenhos elou fluxograma para reforçar meu aprendizado) tiveram $47 \%$ que concordaram totalmente contra nenhum discordante totalmente, os alunos da aprendizagem colaborativa, perceberam como o uso de uma estratégia metodológica facilitou para que alcançassem melhores resultados.

No aspecto comportamental encontram-se as questões 15 e 16. Na afirmativa 15 (Após a monitoria aprendi a planejar melhor meu estudo) tiveram $44 \%$ participantes concordando totalmente e nenhum discordando totalmente. Ao fazer um planejamento de estudo os alunos se envolvem no processo de aprendizagem, programam-se quanto ao material de pesquisa, criam critérios de relevância quanto o conteúdo, organizam o tempo e estabelecem uma complexidade crescente da construção do conhecimento. A afirmativa 15 é validada com a 16 (Antes das monitorias eu não tinha um planejamento de estudo), tendo aqui $26 \%$ concordado parcialmente e $26 \%$ concordado totalmente, o que enfatiza um total de $52 \%$ alunos que não possuíam um planejamento eficaz de estudo para este componente curricular.

"Sempre tive uma facilidade de oratória, cheguei a dar monitoria nos anos de 2018 e 2019, sempre ia nas monitorias das matérias que cursava, sempre fui organizada com os estudos mas digo pelo o que observo dos outros colegas e dos que foram meus alunos, aqueles que não se organizavam tão bem passaram a entender melhor a importância disso" (Fala do aluno entrevistado na afirmativa 24).

A afirmativa 21 (Eu recomendaria que esse metodo de monitoria colaborativa fosse aplicado em outros componentes curriculares) teve o intuito de avaliar se houve a compreensão dos alunos sobre a metodologia colaborativa. Foi observado que $95 \%$ dos entrevistados concordaram totalmente e 5\% concordado parcialmente, não havendo nenhum discordante. Sendo esta a afirmativa com maior aceitabilidade, com isso infere-se que os alunos tiveram boa adesão ao método e compreenderam a importância deste. 
O conteúdo do estudo ativo seguiu o cronograma das aulas administradas tradicionalmente, com estudo da anatomia sistêmica. Todo os sistemas anatômicos foram abordados em um semestre e ao final deste foi aplicado um questionário. A pesquisa empregou a escala de Likert de cinco pontos, com a seguinte análise: 1 = Concordo totalmente: concorda em $100 \%$ da afirmativa; 2 = Concordo parcialmente: concorda com a afirmativa, mas não em 100\%; 3 = Sem condições de opinar; $4=$ Discordo parcialmente: discorda da afirmativa, mas não em 100\%; 5 = Discordo totalmente: discorda em $100 \%$ da afirmativa. Likert é empregada em pesquisas de opinião, na qual os entrevistados especificam o seu nível de concordância com a afirmação.

A partir da afirmativa subjetiva do item 24 (Posso dizer que a monitoria executada na forma colaborativo despertou em mim) pode-se inferir mais sobre a percepção dos alunos sobre as monitorias ministrada na forma de aprendizagem colaborativa (Tabela 6).

Tabela 6 - Relato de experiência dos docentes atuantes nas monitorias colaborativa

\section{Esperado}

1) Empoderamento discente

2) Melhor relação com seus pares

\section{Relato de experiência}

do • "Uma ótima experiência, melhorou muito o meu desempenho e a forma como foram criados laços com as minhas colegas de grupo"

- "Me despertou o senso de ajudar o próximo"

- "É cada dia de monitoria uma troca de experiências entre alunos, o que deixa o ambiente muito mais agradável por todos estarem no mesmo barco. Logo você se imagina fazendo o mesmo que aquele veterano que está ali te ajudando"

- "Uma nova forma de estudar, menos centralizada"

- “É gratificante ajudar uma outra pessoa, porque é muito bom quando somos ajudados também, a monitoria tem essa recíproca, você ajuda e é ajudado"

3) Despertar para docência - "Interesse em ser monitora"

4) Melhorar a oratória - "Maior atratividade para docência"

- "Interesse em ser monitora, interesse em levar esse conhecimento a comunidade para que todos pudessem ter acesso"

- "O interesse em ser monitora e utilizar e modificar um pouco o metodo utilizado"

5) Melhorar o rendimento • "Uma motivação para estudar mais e me ajudou a não desanimar quando tinha que acadêmico estudar um conteúdo mais complicado"

6) Aumentar o interesse do aluno por este componente curricular

- "Mais interesse sobre a matéria"

- "Interesse pela área"

- "Vontade de estudar mais ainda Anatomia"

- "Mais interesse sobre a matéria"

- "Grande vontade de repassar esse método para outras pessoas, como fazemos no projeto de extensão de visitas aos laboratórios da saúde"

7) Torna-los cognitivamente ativos, despertando-os para sua autonomia

- "Maior interesse e eficiência na matéria estudada"

- "Interesse e motivação"

- "É super interessante como as monitorias ajudam você a despertar a vontade em aprender cada vez mais a disciplina"

- "Disciplina e foco para organizar os conteúdos de uma forma que eu conseguisse assimilar e revisar o máximo possível"

- "Vontade de buscar conhecimento, de aprender mais, não apenas na matéria de anatomia, mas em outras disciplinas também"

Fonte: Dados Trabalhados pelos autores.

Através do diário de notas da disciplina de Estudos Morfológicos Macroscópicos (Anatomia Humana) dos cursos de Enfermagem e Nutrição foi possível verificar o rendimento dos discentes, concluindo que em todas as turmas o grupo de discentes participantes da metodologia colaborativa apresentaram resultados superiores à média e ao grupo de discentes não participantes em relação as notas (Tabela 7). 
Tabela 7 - Relação de notas médias de turmas de Enfermagem e Nutrição.

\begin{tabular}{|c|c|c|c|c|c|}
\hline \multicolumn{2}{|c|}{} & \multicolumn{3}{|c|}{ Nota média } \\
\hline Ano & Semestre & Curso & Participantes & Não participantes & Geral \\
\hline 2017 & $1^{\circ}$ & Enfermagem & 8,6 & 2,6 & 5,6 \\
\hline 2017 & $1^{\circ}$ & Nutrição & 6,9 & 2,9 & 4,4 \\
\hline 2017 & $2^{\circ}$ & Enfermagem & 6,9 & 3,4 & 5,4 \\
\hline 2017 & $2^{\circ}$ & Nutrição & 6,1 & 4,3 & 4,9 \\
\hline 2018 & $1^{\circ}$ & Enfermagem & 7,5 & 2,9 & 5,5 \\
\hline 2018 & $2^{\circ}$ & Nutrição & 6,9 & 2,8 & 4,4 \\
\hline
\end{tabular}

Fonte: Dados Trabalhados pelos autores.

Chega-se à conclusão de que o emprego de prova, avaliação, testes e notas são ferramentas válidas, sendo quase que inevitável o uso desse "incentivo" na nossa cultura competitiva. Ausubel (2003) lembra que o processo produtivo deve preferir o trabalho em equipe, mas deve haver também momentos individualizados, nos quais seja possível avaliar a condição de cada estudante. Os defensores da aprendizagem assistida por pares sugerem que ela é bem-sucedida porque professor e aluno compartilham uma base de conhecimento semelhante, ou seja, um "conhecimento cognitivo congruente" (Lockspeiser et al., 2008).

Ao analisar os anos letivos observa-se que existe uma maior adesão da turma de nutrição à monitoria colaborativa, em relação ao total de participantes, com percentual de adesão de $26 \%$ do total (Gráfico 2).

Gráfico 2 - Percentual de participação em metodologia colaborativa.

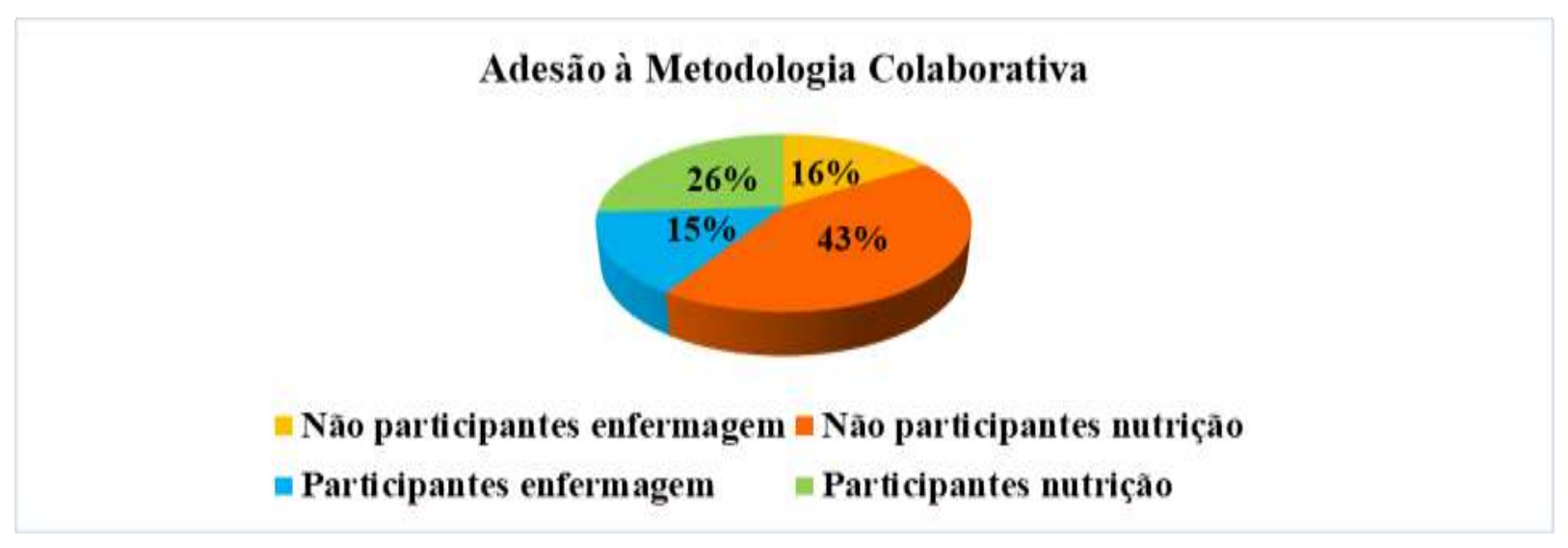

Fonte: Dados Trabalhados pelos autores.

Quanto ao rendimento acadêmico observou-se que no período letivo de 2017.1 e 2018.1 houveram $100 \%$ de aprovação dos acadêmicos de enfermagem que participaram da monitoria colaborativa, contra uma reprovação de $63 \%$ dos não participantes (Tabela 8). Já no curso de nutrição somente no período de 2018.1 houve $100 \%$ de aprovação, em 2017.1 dos participantes da monitoria colaborativa ainda houveram $12 \%$ de reprovação e $31 \%$ em 2017.2, este semestre correspondeu ao período letivo de menos adesão dos alunos do curso de nutrição ao método (36\%). 
Tabela 8 - Percentuais de Aprovação e Reprovação.

\begin{tabular}{|l|c|c|c|c|}
\hline \multicolumn{2}{|c|}{ Participantes da Monitoria } & \multicolumn{2}{c|}{ Não participantes da Monitoria } \\
\hline \multicolumn{1}{|c|}{ Turma } & Aprovação & Reprovação & Aprovação & Reprovação \\
\hline Enfermagem 2017.1 & $100 \%$ & $0 \%$ & $37 \%$ & $63 \%$ \\
\hline Enfermagem 2017.2 & $82 \%$ & $18 \%$ & $37 \%$ & $63 \%$ \\
\hline Enfermagem 2018.1 & $100 \%$ & $0 \%$ & $46 \%$ & $54 \%$ \\
\hline Nutrição 2017.1 & $88 \%$ & $12 \%$ & $33 \%$ & $67 \%$ \\
\hline Nutrição 2017.2 & $69 \%$ & $31 \%$ & $64 \%$ & $36 \%$ \\
\hline Nutriçãão 2018.1 & $100 \%$ & $0 \%$ & $36 \%$ & $64 \%$ \\
\hline
\end{tabular}

Fonte: Dados Trabalhados pelos autores.

A partir da interpretação do coeficiente de variação em relação a variabilidade das notas nota-se que em amostras de acadêmicos não participantes da metodologia colaborativa alguns grupos apresentaram variabilidade maior que 100\%, o que indica alta dispersão de dados, indicando assim que houve um desvio padrão superior a média. Os discentes participantes do curso de Enfermagem 2017.1 obtiveram notas com variação de 8\%, o que indica que este grupo apresentou maior homogeneidade ou baixa dispersão (Tabela 9).

Tabela 9 - Coeficiente de Variação (\%) em Relação a Adesão da Metodologia Colaborativa.

\begin{tabular}{|c|c|c|c|c|}
\hline \multirow{2}{*}{$\begin{array}{c}\text { Ano/ } \\
\text { Semestre }\end{array}$} & \multicolumn{2}{|c|}{ Enfermagem } & \multicolumn{2}{c|}{ Nutrição } \\
\hline $\mathbf{2 0 1 7 . 1}$ & Participantes & Não Participantes & Participantes & Não Participantes \\
\hline $\mathbf{2 0 1 7 . 2}$ & $3 \%$ & $138 \%$ & $28 \%$ & $102 \%$ \\
\hline $\mathbf{2 0 1 8 . 1}$ & $13 \%$ & $80 \%$ & $44 \%$ & $68 \%$ \\
\hline
\end{tabular}

Fonte: Dados Trabalhados pelos autores.

Com os resultados satisfatórios pode-se inferir que os alunos alcançaram o proposto, assumir a responsabilidade adequada pela sua própria aprendizagem e tornar-se ativo cognitivamente. Que segundo Ausubel (2003, p.52) se concretiza quando o estudante:

1. Aceita a tarefa de aprender ativamente, procurando compreender o material de instrução que the ensinam.

2. Tenta, de forma genuína, integrá-lo nos conhecimentos que já possui.

3. Não evita o esforço ou batalha por novas aprendizagens difíceis e não exige que o professor 'lhe faça a papa toda'.

4. Decide fazer as perguntas necessárias sobre o que não compreende.

\section{Conclusão}

Conclui-se que o implemento de metodos ativos, como o colaborativo, se tornam eficazes tão logo que são aceito pelos alunos. Pois, oferece um feedback do que se aprende, proporcionando a interação, autorregulação, empoderamento, autonomia, rendimento e sobretudo o pensamento crítico. Aos alunos que participaram do metodo proposto na pesquisa observou-se um maior índice de aprovação, tendo algumas monitorias colaborativas resultado em $100 \%$ de aprovação do grupo (exemplificado na Tabela 8). Ademais 95\% (dp 0,64) dos participantes recomendariam a aplicação do metodo em outros componentes curriculares e $92 \%$ (dp 0,59) afirmam que conseguiram associar melhor o conteúdo teórico com o prático com o uso das monitorias colaborativas.

O uso de metodologias ativas, na forma colaborativa, aplicada de forma conjunta com o método tradicional na disciplina de Anatomia Humana ancora novos ensinamentos de forma não-arbitraria e substantiva com as ideias já existentes 
dos alunos, saindo de um aprendizado mecânico para um significativo. Os estudantes apontaram que a monitoria na forma colaborativa permitiu melhor compreensão e interesse no estudo dos conteúdos e que o estudo em grupo favoreceu a motivação, o auxílio e o fortalecimento das relações com os colegas. Diante dos grandes desafios enfrentados no ensino de anatomia, o estímulo para execução da monitoria na forma colaborativa pode ser uma ferramenta para auxiliar nas estratégias de ensino.

\section{Referências}

Almeida, N. D. F., Sousa, R. D. D., Moura, M. C., Silva, T. D., \& Strini, P. J. S. (2015). Avaliação da importância e do aprendizado promovido em atividades de Extensão Universitária. Revista UFG, ano XV, n. 17.

Ausubel, D. P. (2003). Aquisição e retenção de conhecimentos: uma perspectiva cognitiva. Plátano, 1.

Aversi-ferreira, T. A., Lopes, D. B., Dos reis, S. M. M., De abreu, T., De freitas aversi-ferreira, G. M., Vera, I., \& Lucchese, R. (2017). Practice of dissection as teaching methodology in anatomy for nursing education. Journal of Morphological Sciences, 26 (3-4).

Bellan, Z. (2008). Heutagogia: aprenda a aprender mais e melhor. SOCEP Editora: Santa Bárbara do Oeste-SP.

Bergman, E. M., Sieben, J. M., Smailbegovic, I., de Bruin, A. B., Scherpbier, A. J., \& van der Vleuten, C. P. (2013). Constructive, collaborative, contextual, and self-directed learning in surface anatomy education. Anatomical sciences education, 6(2), 114-124.

Bergman, E. M., Sieben, J. M., Smailbegovic, I., de Bruin, A. B., Scherpbier, A. J., \& van der Vleuten, C. P. (2013). Constructive, collaborative, contextual, and self-directed learning in surface anatomy education. Anatomical sciences education, 6(2), 114-124.

Braz, P. R. P. (2009). Método didático aplicado ao ensino da anatomia humana.

Carvalho, C. A. F. (2017). Utilização de Metodologia Ativa de Ensino nas Aulas Práticas de Anatomia. Revista de Graduação USP, 2(3), 117-121.

Da Silva, E. V., Aversi-Ferreira, T. A., da Rocha, A. O., de Sousa Leite, K. J. N., Faria, A. B., da Silva, A. D. D., \& de Abreu, T. (2020). Body Donation Programs in Brazil. Brazilian Journal of Development, 6(12), 101260-101271.

Da Veiga Simão, A. M., \& Frison, L. M. B. (2013). Autorregulação da aprendizagem: abordagens teóricas e desafios para as práticas em contextos educativos. Cadernos de Educação, 5, p. 02-20.

De Castro, S. K. A., Nishijo, H., \& Aversi-ferreira, T. A. (2018). Neuroanatomy teaching: an example of active teaching applied to medical formation. American Journal of Educational Research and Reviews, 3, 37-37.

De Sousa, G. D. C. D., \& Junior, T. D. A. A. (2017). A importância da monitoria acadêmica no processo ensino-aprendizagem em Fisiologia Humana. Encontro de Extensão, Docência e Iniciação Científica (EEDIC), 3(1).

Ertmer, P. A., \& Newby, T. J. (1993). Behaviorism, cognitivism, constructivism: Comparing critical features form an instructional design perspective. Perform Improv, 6, 50-72.

Fornaziero, C. C., Gordan, P. A., Carvalho, M. A. V. D., Araujo, J. C., \& Aquino, J. C. B. D. (2010). O Ensino da Anatomia: Integração do Corpo Humano e Meio Ambiente. Revista brasileira de educação médica, Brasília, 34(2), 290-297.

Gardner, E., J Gray, D., \& O'Rahilly, R. (1963). Anatomy: a regional study of human structure. WB Saunders.

Gokhale, A. A. (1995). Collaborative learning enhances critical thinking.

Hase, S \& Kenyon, C. (2011). Retrieved from From Andragogy to Heutagogy. February. Disponível em: http://ultibase.rmit.edu.au/Articles/dec00/hase2.htm

Kerby, J., Shukur, Z. N., \& Shalhoub, J. (2011). The relationships between learning outcomes and methods of teaching anatomy as perceived by medical students. Clinical Anatomy, 24 (4), 489-497.

Leung, K. K., Lu, K. S., Huang, T. S., \& Hsieh, B. S. (2006). Anatomy instruction in medical schools: connecting the past and the future. Advances in Health Sciences Education, 11(2), 209-215.

Lockspeiser, T. M., O’Sullivan, P., Teherani, A., \& Muller, J. (2008). Understanding the experience of being taught by peers: the value of social and cognitive congruence. Advances in Health Sciences Education, 13(3), 361-372.

Longhurst, G. J., Stone, D. M., Dulohery, K., Scully, D., Campbell, T., \& Smith, C. F. (2020). Strength, weakness, opportunity, threat (SWOT) analysis of the adaptations to anatomical education in the United Kingdom and Republic of Ireland in response to the Covid-19 pandemic. Anatomical sciences education, 13(3), 301-311.

Mclachlan, J. C., \& Patten, D. (2006). Anatomy teaching: ghosts of the past, present and future. Medical education, 40 (3), $243-253$.

Mitre, S. M., Siqueira-Batista, R., Girardi-de-Mendonça, J. M., Morais-Pinto, N. M. D., Meirelles, C. D. A. B., Pinto-Porto, C., \& Hoffmann, L. M. A. (2008). Metodologias ativas de ensino-aprendizagem na formação profissional em saúde: debates atuais. Ciência \& saúde coletiva, 13, $2133-2144$.

Montalvo, F. T., \& Torres, M. G. (2004). Self-regulated learning: Current and future directions. 
Research, Society and Development, v. 10, n. 4, e13410413885, 2021

(CC BY 4.0) | ISSN 2525-3409 | DOI: http://dx.doi.org/10.33448/rsd-v10i4.13885

Moxham, B. J., \& Moxham, S. A. (2021). The relationships between attitudes, course aims and teaching methods for the teaching of gross anatomy in the medical curriculum. European Journal of Anatomy, 11(S1), 19-30.

Nóbrega, K. S. D. A., \& Tavares, R. (2008). A aprendizagem de anatomia: A utilização de mapas conceituais como uma ferramenta instrucional. XXII Congresso Brasileiro de Anatomia, Belém, outubro.

Patel, K. M., \& Moxham, B. J. (2008). The relationships between learning outcomes and methods of teaching anatomy as perceived by professional anatomists. Clinical Anatomy: The Official Journal of the American Association of Clinical Anatomists and the British Association of Clinical Anatomists, 21(2), 182-189.

Pereira, A. T. C., Schmitt, V., \& Dias, M. R. A. C. (2007). Ambientes virtuais de aprendizagem. AVA-Ambientes Virtuais de Aprendizagem em Diferentes Contextos. Rio de Janeiro: Editora Ciência Moderna Ltda, 4(22).

Pfrimer, G. D. A., De abreu, T., Vieira, V. S., Soares, N. P., Aversi-ferreira, R. A. G. M. F., gratão, L. H. A., \& Aversi-ferreira, T. A. (2012). Historic and teaching aspects of anatomy and cebus genus role in contemporary anatomy. International Journal of Morphology, 30(2), 607-612.

Regehr, G., Norman, G. R. (1996). Issues in cognitive psychology: Implications for Educação profissional. Acad Med, 71, 988-1001.

Salbego, C., Oliveira, E. M. D. D., Silva, M. D. A. R. D., \& Bugança, P. R. (2015). Percepções acadêmicas sobre o ensino e a aprendizagem em anatomia humana. Revista Brasileira de Educação Médica, 39(1), 23-31.

Schiffman, L. G., \& Kanuk, L. L. (2000). Comportamiento del consumidor. Pearson educación.

Souza Júnior, I. (2010). Métodos de ensino aprendizagem em anatomia humana: primeira etapa do programa institucional de bolsas acadêmicas (PIBAC) do IFPI/Campus Floriano. In Campus Floriano. Apresentação de Trabalho/Congresso.

Standring, S., Ellis, H., Healy, J., Johnson, D., Williams, A., Collins, P., \& Wigley, C. (2005). Gray's anatomy: the anatomical basis of clinical practice. American journal of neuroradiology, 26(10), 2703.

Sugand, K., Abrahams, P., \& Khurana, A. (2010). The anatomy of anatomy: a review for its modernization. Anatomical sciences education, 3(2), 83-93.

Tanasi, C. M., Tanase, V. I., \& Harsovescu, T. (2014). Modern methods used in the study of human anatomy. Procedia-Social and Behavioral Sciences, 127, 676-680.

Tiznado-Matzner, G., Bucarey-Arriagada, S., \& Lizama-Pérez, R. (2020). Modelos Virtuales Tridimensionales de Muestras Cadavéricas Reales Obtenidas con Escáner 3D, Utilizados como Recurso Educativo Complementario para el Estudio de la Anatomía Humana: Percepción de los Estudiantes Universitarios Enfrentados a esta Nueva Tecnología. International Journal of Morphology, 38(6), 1686-1692.

Torres, P. L., \& Irala, E. A. F. (2014). Aprendizagem colaborativa: teoria e prática. Complexidade: redes e conexões na produção do conhecimento. Curitiba: Senar, 61-93.

Vygotsky, L. S. (2012). The collected works of LS Vygotsky: The fundamentals of defectology (abnormal psychology and learning disabilities). Springer Science \& Business Media. 